\title{
Cluster analysis of DCE-MRI data identifies regional tracer- kinetic changes after tumor treatment with high intensity focused ultrasound
}

\author{
Citation for published version (APA): \\ Jacobs, I., Hectors, S. J. C. G., Schabel, M. C., Grull, H., Strijkers, G. J., \& Nicolay, K. (2015). Cluster analysis of \\ DCE-MRI data identifies regional tracer-kinetic changes after tumor treatment with high intensity focused \\ ultrasound. NMR in Biomedicine, 28(11), 1443-1454. https://doi.org/10.1002/nbm.3406
}

DOI:

10.1002/nbm.3406

Document status and date:

Published: 01/11/2015

\section{Document Version:}

Publisher's PDF, also known as Version of Record (includes final page, issue and volume numbers)

\section{Please check the document version of this publication:}

- A submitted manuscript is the version of the article upon submission and before peer-review. There can be important differences between the submitted version and the official published version of record. People interested in the research are advised to contact the author for the final version of the publication, or visit the $\mathrm{DOI}$ to the publisher's website.

- The final author version and the galley proof are versions of the publication after peer review.

- The final published version features the final layout of the paper including the volume, issue and page numbers.

Link to publication

\footnotetext{
General rights

- You may freely distribute the URL identifying the publication in the public portal. follow below link for the End User Agreement:

www.tue.nl/taverne

Take down policy

If you believe that this document breaches copyright please contact us at:

openaccess@tue.nl

providing details and we will investigate your claim.
}

Copyright and moral rights for the publications made accessible in the public portal are retained by the authors and/or other copyright owners and it is a condition of accessing publications that users recognise and abide by the legal requirements associated with these rights.

- Users may download and print one copy of any publication from the public portal for the purpose of private study or research.

- You may not further distribute the material or use it for any profit-making activity or commercial gain

If the publication is distributed under the terms of Article $25 \mathrm{fa}$ of the Dutch Copyright Act, indicated by the "Taverne" license above, please 


\title{
Cluster analysis of DCE-MRI data identifies regional tracer-kinetic changes after tumor treatment with high intensity focused ultrasound
}

\author{
Igor Jacobs ${ }^{a *,+}$, Stefanie J. C. G. Hectors ${ }^{a, b \dagger}$, Matthias C. Schabel ${ }^{c, d}$, \\ Holger Grüll ${ }^{a, e}$, Gustav J. Strijkers ${ }^{a, f}$ and Klaas Nicolay ${ }^{a}$
}

\begin{abstract}
Evaluation of high intensity focused ultrasound (HIFU) treatment with MRI is generally based on assessment of the non-perfused volume from contrast-enhanced $T_{1}$-weighted images. However, the vascular status of tissue surrounding the non-perfused volume has not been extensively investigated with MRI. In this study, cluster analysis of the transfer constant $K^{\text {trans }}$ and extravascular extracellular volume fraction $v_{e}$, derived from dynamic contrastenhanced MRI (DCE-MRI) data, was performed in tumor tissue surrounding the non-perfused volume to identify tumor subregions with distinct contrast agent uptake kinetics.

DCE-MRI was performed in CT26.WT colon carcinoma-bearing BALB/c mice before $(n=12)$, directly after $(n=12)$ and 3 days after $(n=6)$ partial tumor treatment with HIFU. In addition, a non-treated control group $(n=6)$ was included. The non-perfused volume was identified based on the level of contrast enhancement. Quantitative comparison between non-perfused tumor fractions and non-viable tumor fractions derived from NADH-diaphorase histology showed a stronger agreement between these fractions 3 days after treatment $\left(R^{2}\right.$ to line of identity $=$ $0.91)$ compared with directly after treatment $\left(R^{2}=0.74\right)$. Next, $k$-means clustering with four clusters was applied to $K^{\text {trans }}$ and $v_{\mathrm{e}}$ parameter values of all significantly enhanced pixels. The fraction of pixels within two clusters, characterized by a low $K^{\text {trans }}$ and either a low or high $v_{\mathrm{e}}$, significantly increased after HIFU. Changes in composition of these clusters were considered to be HIFU induced. Qualitative H\&E histology showed that HIFU-induced alterations in these clusters may be associated with hemorrhage and structural tissue disruption. Combined microvasculature and hypoxia staining suggested that these tissue changes may affect blood vessel functionality and thereby tumor oxygenation. In conclusion, it was demonstrated that, in addition to assessment of the non-perfused tumor volume, the presented methodology gives further insight into HIFU-induced effects on tumor vascular status. This method may aid in assessment of the consequences of vascular alterations for the fate of the tissue. Copyright $\odot 2015$ John Wiley \& Sons, Ltd.
\end{abstract}

Additional supporting information may be found in the online version of this article at the publisher's web site.

Keywords: dynamic contrast-enhanced MRI; cluster analysis; high intensity focused ultrasound; cancer treatment response

\section{INTRODUCTION}

High intensity focused ultrasound (HIFU) is an emerging technique for the non-invasive thermal treatment of tumors (1). While routine clinical use of HIFU treatment is mainly restricted

\footnotetext{
* Correspondence to: I. Jacobs, Biomedical NMR, Department of Biomedical Engineering, Eindhoven University of Technology, Eindhoven, 5600 MB, The Netherlands.

E-mail: i.jacobs@tue.nl

a I. Jacobs, S. J. C. G. Hectors, H. Grüll, G. J. Strijkers, K. Nicolay Biomedical NMR, Department of Biomedical Engineering, Eindhoven University of Technology, Eindhoven, The Netherlands

b S. J. C. G. Hectors

Translational and Molecular Imaging Institute, Icahn School of Medicine at Mount Sinai, New York, NY, USA

c M. C. Schabel

Imaging Research Center, Oregon Health and Science University, Portland, OR, USA
}

to non-cancerous growths, such as uterine fibroids (2-4), its application range is now being extended to malignant tumors, such as breast $(5,6)$, prostate (7-10) and liver cancer (11-13).
d M. C. Schabel
Utah Center for Advanced Imaging Research, University of Utah, Salt Lake City, UT, USA
e H. Grüll
Oncology solutions, Philips Research, Eindhoven, The Netherlands

\section{f G. J. Strijkers}
Biomedical Engineering and Physics, Academic Medical Center, University of Amsterdam, Amsterdam, The Netherlands
+ These authors contributed equally.

Abbreviations used: AlF, arterial input function; $C A$, contrast agent; DCE$M R I$, dynamic contrast-enhanced MRI; EPl, echo planar imaging; H\&E, hematoxylin and eosin; HIFU, high intensity focused ultrasound; NADH, nicotinamide adenine dinucleotide; $R O I$, region of interest; $S D$, standard deviation. 
Ultrasound-guided and MRI-guided HIFU (MR-HIFU) systems have been developed that provide spatial guidance of the treatment (1,14-16). In addition, an MR-HIFU system facilitates treatment monitoring using MR thermometry and can be exploited for assessment of treatment outcome (17-19).

Contrast-enhanced $T_{1}$-weighted imaging is one of the most commonly used MRI methods for the evaluation of HIFU treatment $(8,9,20)$. A lack of contrast enhancement is generally observed in the central ablation volume, which is exposed to temperatures of approximately $60{ }^{\circ} \mathrm{C}$ during treatment. The region with low contrast enhancement is referred to as the nonperfused volume, and serves as an important readout parameter for HIFU treatment evaluation (9,20-22). In the transition zone between the central ablation volume and unaffected surrounding tissue, milder temperature elevations may result in indirect heat-induced injury and more subtle vascular alterations. The vascular changes in this peripheral zone are strongly dependent on the exact temperature and/or thermal dose that is reached during the HIFU intervention and can be transient in nature. Directly after HIFU, hyperthermic conditions in the peripheral zone could (transiently) increase tumor blood flow and microvascular permeability and increase tumor oxygenation $(23,24)$. Within a few days after treatment, the immune response may be enhanced in the transition zone and inflammation-associated hyperemia may be observed $(8,20,25)$. At a later stage (approximately 7 days (26)) after treatment, fibrosis and revascularization may also alter the tumor vascular status (26-28). On contrastenhanced $T_{1}$-weighted images, an enhancing rim is often reported in the region surrounding the central ablation zone, which may be due to these vascular alterations or result from residual unaffected tumor tissue in the case of incomplete ablation $(8,20)$. Since the observed contrast enhancement is almost equal in regions of different vascular changes and in unaffected residual tumor tissue, conventional contrast-enhanced MRI does not have a high specificity for the identification of subtle vascular effects after HIFU treatment.

It is however important to gain further insights into the vascular status of the peripheral tumor zone, since HIFU-induced changes in the microvascular function of the tumor tissue in this region may have substantial consequences for the microenvironment and fate of the tumor. In addition, if residual tumor is present after HIFU treatment, this may be treated with adjuvant tumor therapies. The sensitivity of the tumor to these therapies may also be affected by the vascular status (25).

Compared with conventional contrast-enhanced MRI, assessment of changes in contrast-agent uptake kinetics by dynamic contrast-enhanced MRI (DCE-MRI) may be a more suitable method to gain insight into the altered vascular status in the tissue around the coagulated lesion. Pharmacokinetic modeling of DCE-MRI data can be applied to determine biomarkers that are sensitive to vascular changes; e.g., with the widely employed Tofts model the transfer constant $K^{\text {trans }}$ and the extravascular extracellular volume fraction $v_{\mathrm{e}}$ can be determined (29). Hijnen et al. have used these kinetic parameters for HIFU therapy assessment in a rat tumor model (30). Cheng et al. have shown that these parameters could give insight into regional changes in the microvasculature after HIFU treatment of rabbit muscle tissue $(26,31)$. However, there are no studies in which the anticipated subtle changes in tumor vascular status around the HIFU ablation volume have been investigated with pharmacokinetic modeling of DCE-MRI data, while knowledge of changes in DCE-MRI-derived kinetic parameters may provide a more refined picture of the tumor vascular status after HIFU treatment compared with conventional contrast-enhanced MRI.

To address this, in the present study, DCE-MRI was performed before, directly after and 3 days after HIFU treatment of a murine subcutaneous tumor model. Partial tumor treatment was performed to allow for the presence of both HIFU-treated and residual tumor tissue after the HIFU procedure. Tracer-kinetic modeling was performed on the tumor pixels outside the nonperfused volume to assess HIFU-induced changes in contrast agent uptake kinetics. Cluster analysis based on $K^{\text {trans }}$ and $v_{\mathrm{e}}$ was employed to identify subregions with different uptake kinetics, and the regional distribution of these clusters within the tumor was determined.

\section{EXPERIMENT}

\section{Ethics statement}

All animal experiments were performed according to Directive 2010/63/EU of the European Parliament and approved by the Animal Care and Use Committee of Maastricht University (protocols 2010-097 and 2010-132).

\section{Murine tumor model}

10-12 week-old BALB/c mice (Charles River, Maastricht, The Netherlands) were inoculated with $2 \times 10^{6}$ CT26.WT murine colon carcinoma cells (American Type Culture Collection (ATCC), CRL-2638; early passages (5-10)), subcutaneously in the right hind limb. The tumors were grown in the hind limb to prevent potential HIFU-induced damage to vital organs and respiration artifacts in the MR measurements. Approximately 10 days after inoculation, tumors became palpable in all animals.

\section{Study design}

MRI was performed 1 day before $(n=12)$, directly $(2-3$ h) after $(n=12)$ and 3 days after HIFU treatment $(n=6)$. A control group of non-treated animals ( $n=6)$ was included that underwent MRI at the same time points as the HIFU-treated animals. The time points of the control animals are referred to as day -1 , day 0 and day 3. Directly after the last MRI experiment the mice were sacrificed, and the tumors were excised for histological analysis. This study design led to three different groups for quantitative histology: animals sacrificed after the MRI examination directly after HIFU treatment ( $n=6$, referred to as "directly after HIFU"), animals sacrificed after the MRI examination 3 days after HIFU treatment ( $n=6$, referred to as " 3 days after HIFU") and nontreated control animals ( $n=6$, referred to as "control").

\section{HIFU treatment}

HIFU treatment was performed outside the MR scanner with the preclinical Therapy Imaging Probe System (TIPS, Philips Research, Briarcliff Manor, NY, USA) (32). Treatment was performed under general anesthesia (1-2\% isoflurane) and precautionary analgesia (buprenorphine, $0.1 \mathrm{mg} / \mathrm{kg}$ s.c., administered at least $30 \mathrm{~min}$ before treatment). Control animals received an equal dose of analgesia at the corresponding time point. Partial tumor treatment was performed by definition of a square $4 \times 4 \mathrm{~mm}^{2}$ treatment grid within the tumor area, consisting of 25 equally spaced ablation volumes $\left(1 \times 1 \times 9 \mathrm{~mm}^{3}\right)$ that were placed within the tumor under guidance of an ultrasound imaging 
system (HDI5000 imaging system combined with a P7-4 phased array transducer, Philips Ultrasound, Bothell, WA, USA). This resulted in the presence of both HIFU-treated and non-treated tumor tissue after the HIFU procedure. A schematic drawing of the HIFU treatment set-up is shown in Supplementary Figure S1. Treatment settings were the following: frequency 1.4 $\mathrm{MHz}$, pulse repetition frequency $20 \mathrm{~Hz}$, acoustic power $12 \mathrm{~W}$, duty cycle $50 \%$, and treatment time $30 \mathrm{~s}$. A wait time of $120 \mathrm{~s}$ was applied between sonication of consecutive treatment points to allow for sufficient cooling of the treated tissue. A temperature increase to approximately $66^{\circ} \mathrm{C}$ during HIFU treatment was confirmed by insertion of a thermocouple into the tumor in three pilot experiments, as previously described (33).

\section{MRI measurements}

MRI measurements were made with a 6.3 T horizontal-bore scanner (Bruker BioSpin, Ettlingen, Germany) using a $3.2 \mathrm{~cm}$ diameter quadrature birdcage RF coil (Rapid Biomedical, Rimpar, Germany) under general anesthesia (1-2\% isoflurane). The mice were positioned in a custom-made cradle, equipped with a mask for anesthetic gas. Respiration was monitored with a balloon sensor. Body temperature was monitored and maintained with a warm water pad. For reduction of susceptibility artifacts in echo planar imaging (EPI), the tumor-bearing paw was covered with degassed ultrasound gel (Aquasonic ${ }^{\circledR}$ 100, Parker Laboratories, Fairfield, NJ, USA). Artifacts were further reduced by local shimming.

A fat-suppressed $T_{2}$-weighted spin-echo sequence $\left(T_{\mathrm{E}}=30 \mathrm{~ms}\right.$, $T_{\mathrm{R}}=1000 \mathrm{~ms}$, number of averages, NA $=1$ ) was used for anatomical reference.

Pre-contrast $T_{1}$ mapping was performed using an inversion recovery Look-Locker EPI sequence $\left(T_{E}=8 \mathrm{~ms}, T_{\mathrm{R}}=10000 \mathrm{~ms}\right.$, inversion time $=30 \mathrm{~ms}$, flip angle $=20^{\circ}$, pulse separation $=400 \mathrm{~ms}$, number of points $=15, \mathrm{NA}=2$ ).

DCE-MRI measurements were made using a gradient-spoiled dual gradient echo EPI sequence $\left(T_{\mathrm{R}}=1250 \mathrm{~ms}, T_{\mathrm{E} 1} / T_{\mathrm{E} 2}=7.5 /\right.$ $26.1 \mathrm{~ms}, \mathrm{NA}=2$, number of segments $=2$, flip angle $=80^{\circ}$, temporal resolution $=5 \mathrm{~s}$, number of repetitions $=100$ ). At $1.5 \mathrm{~min}$ after start of the acquisition, a bolus of $0.3 \mathrm{mmol} / \mathrm{kg}$ Gd-DOTA (Dotarem; Guerbet, Villepinte, France) with a saline flush was injected in $5 \mathrm{~s}$ via the tail vein using an infusion pump (Chemyx Fusion 100, Stafford, TX, USA).

All images were acquired with a matrix size of $128 \times 128$, FOV of $4 \times 4 \mathrm{~cm}^{2}$ and $1 \mathrm{~mm}$ slice thickness. 12-16 slices were acquired, covering the whole tumor volume.

\section{DCE-MRI data processing}

Image analysis was performed in Mathematica 8.0 (Wolfram Research, Champaign, IL, USA) and MATLAB R2013a (MathWorks, Natick, MA, USA). Regions of interest (ROIs) were defined on the $T_{2}$-weighted images by manually drawing contours around the tumor tissue on each slice. From these ROls the tumor volumes at the different experimental time points were determined. Pre-contrast $T_{1}$ maps were generated as described previously (34).

A $T_{2}{ }^{*}$ correction was performed on the dynamic signal intensities to account for the $T_{2}{ }^{*}$ changes caused by contrast agent influx $(35,36)$. The dynamic $T_{2}{ }^{*}$ values in the tumor pixels were determined based on the ratio of the signal intensities at the two echo times. Dynamic $T_{1}$ values were calculated from the signal equation for a spoiled gradient-echo sequence using the $T_{2}{ }^{*}$-corrected dynamic signal intensities and the pre-contrast $T_{1}$ values. A $B_{1}$ correction was applied to the flip angle used in the dynamic acquisition, using a $B_{1}$ map acquired with the same RF coil in a $2 \%$ agarose $/ 0.05 \mathrm{mM} \mathrm{CuSO}_{4}$ phantom. Dynamic $T_{1}$ values were converted to dynamic contrast agent concentrations ([CA]) using the Dotarem plasma relaxivity at $7 \mathrm{~T}$ and $37^{\circ} \mathrm{C}$ (3.53 $\left.\mathrm{mM}^{-1} \mathrm{~s}^{-1}(37)\right)$.

\section{Pharmacokinetic analysis of DCE-MRI data}

Pharmacokinetic analysis of the DCE-MRI data was performed with a custom-written MATLAB tool. For determination of $K^{\text {trans }}$ and $v_{\mathrm{e}}$ in each tumor pixel, the standard Tofts model $(29,38)$ was used, in which the dynamic CA concentration in the tumor tissue is defined as

$$
C_{\mathrm{t}}(t)=K^{\text {trans }} \int_{0}^{t} C_{\mathrm{p}}(\tau) \mathrm{e}^{-k_{\mathrm{ep}}(t-\tau)} \mathrm{d} \tau
$$

in which $C_{\mathrm{t}}$ and $C_{\mathrm{p}}$ are the CA concentrations in the tumor tissue and the blood plasma, respectively, and $k_{\text {ep }}$ is the rate constant $\left(k_{\mathrm{ep}}=K^{\text {trans }} / v_{\mathrm{e}}\right)$. A delay term $\left(t_{\mathrm{d}}\right)$ was included in the model to allow for a delay between bolus arrival in the blood and the tissue response. A literature-based bi-exponential arterial input function (AIF), measured in the mouse iliac artery upon injection of a similar dose of Dotarem (39), was used and modified to match our data. The bi-exponential AIF is described by

$$
C_{\mathrm{p}}(t)= \begin{cases}0 & t<t_{\mathrm{i}} \\ a_{1} \mathrm{e}^{-m_{1} t}+a_{2} \mathrm{e}^{-m_{2} t} & t \geq t_{\mathrm{i}}\end{cases}
$$

in which $t_{\mathrm{i}}$ is the injection time.

The peak amplitude of the AIF was adapted based on the injected dose and the mouse plasma volume. In addition, the second exponent of the AIF, describing the AIF tail, was adapted based on blood kinetics data of Dotarem from five separate CT26.WT tumor-bearing BALB/C mice. For this purpose, the gadolinium concentration of blood plasma samples acquired at different time points after CA injection was determined by means of inductively coupled plasma-atomic emission spectrometry or inductively coupled plasma-mass spectrometry. These adaptations resulted in the following biexponential AIF parameters: $a_{1}=5.36(\mathrm{mM}), m_{1}=11.20\left(\mathrm{~min}^{-1}\right)$, $a_{2}=1.27(\mathrm{mM}), m_{2}=0.0655\left(\mathrm{~min}^{-1}\right)$.

The standard Tofts model was fitted to the dynamic [CA] curves using the MATLAB function Isqcurvefit, with constraints $K^{\text {trans }} \geq 0 \min ^{-1}, k_{\text {ep }} \geq 0 \min ^{-1}$ and $0 \leq t_{\mathrm{d}} \leq 7$ repetitions.

\section{Identification of non-perfused tumor volume}

Non-perfused pixels were selected based on the level of contrast enhancement. Pixels were considered non-perfused if the median [CA] after injection was lower than five times the standard deviation (SD) of the data points in the dynamic [CA] curve before injection. The non-perfused pixels were not included in the subsequent histogram and cluster analyses. The other tumor pixels are for reasons of simplicity referred to as perfused pixels. It should however be noted that the significant contrast enhancement in these pixels does not necessarily reflect substantial perfusion, since it may also be caused by an enhanced vascular permeability. 


\section{Selection of pixels for cluster analysis}

A number of criteria were defined to select the perfused tumor pixels that were included in the cluster analysis. Pixels in which the dynamic $T_{1}$ became shorter than $250 \mathrm{~ms}$ or the dynamic $T_{2}{ }^{*}$ became shorter than the first echo time $(7.5 \mathrm{~ms})$ were excluded from further analysis, since such low $T_{1}$ and $T_{2}{ }^{*}$ values could not be accurately determined with the present DCE-MRI acquisition settings. Furthermore, pixels for which the fitted curves had a goodness-of-fit $\left(R^{2}\right)$ value lower than 0.8 were excluded from analysis. In addition, pixels with non-physiological $v_{\mathrm{e}}$ values higher than 1 were omitted.

\section{Cluster analysis}

$k$-means clustering with two, three, four, five and six clusters was performed on the combined $K^{\text {trans }}$ and $v_{\mathrm{e}}$ data of the selected perfused pixels of all (HIFU-treated and non-treated) animals at all time points simultaneously with a custom-written Mathematica function. Prior to clustering, data were normalized ( mean $=0, S D=1$ ) to remove scaling differences between $K^{\text {trans }}$ and $v_{\mathrm{e}}$. Changes in the fraction of pixels in the different clusters were defined as treatment associated if the fraction of pixels assigned to the particular cluster increased significantly (onesided paired $t$-test, $p<0.05$ ) after HIFU treatment (either directly or 3 days after HIFU) compared with before HIFU treatment.

\section{Regional distribution of clusters}

To assess the spatial distribution of the different clusters with respect to the non-perfused volume, the distance from each pixel in each cluster to the non-perfused volume was determined. For each pixel in a particular cluster, the distances between that pixel and all non-perfused pixels in the same MRI slice were determined. The minimum of these distances was taken as a measure for the distance between the particular pixel and the nonperfused volume. Histograms (six bins, bin width 1 pixel, range 1-7 pixels) of these minimal distances to the non-perfused volume were made for each cluster at each time point after HIFU treatment to visualize the regional distribution of the different clusters with respect to the non-perfused volume. In addition, the median minimal distance from each cluster to the nonperfused volume was calculated for each HIFU-treated mouse at both time points after HIFU treatment.

\section{Histological analysis}

Dissected tumors were snap-frozen in isopentane and stored at $-80^{\circ} \mathrm{C}$. Tumors were cut into $5 \mu \mathrm{m}$ thick sections with a distance of approximately $300 \mu \mathrm{m}$ between the sections. The cryosections were briefly air-dried and subsequently stained for nicotinamide adenine dinucleotide (NADH) diaphorase activity to assess cell viability. Sections were incubated at $37{ }^{\circ} \mathrm{C}$ for $1 \mathrm{~h}$ in Gomori-Tris- $\mathrm{HCl}$ buffer ( $\mathrm{pH}$ 7.4) containing $\beta-\mathrm{NAD}$ reduced disodium salt hydrate (Sigma-Aldrich, St. Louis, MO, USA, $0.71 \mathrm{mg} / \mathrm{ml}$ buffer solution) and nitro blue tetrazolium (SigmaAldrich, $0.29 \mathrm{mg} / \mathrm{ml}$ buffer solution). Brightfield microscopy was performed on all sections and consisted of mosaic acquisition of the entire section at $5 \times$ magnification. Analysis of the microscopy images was performed in Mathematica 7.0. ROls were manually drawn around the pale non-viable tumor tissue and the entire tumor tissue on all sections of each tumor. From the ratio between the ROI areas of non-viable tumor tissue and entire tumor tissue on all tumor sections, a histology-derived non-viable tumor volume fraction was determined for each tumor. Subsequently, the MRI-derived non-perfused tumor fractions were compared with the histology-derived non-viable tumor fractions.

Apart from the NADH-diaphorase staining, hematoxylin and eosin (H\&E) staining was performed on paraffin sections of a separate CT26.WT tumor that was treated with the same HIFU settings. This tumor was excised at $\sim 2.5 \mathrm{~h}$ after HIFU treatment. Brightfield microscopy was performed to inspect morphological changes in the tumor tissue after treatment.

In order to further interrogate the causes and consequences of HIFU-induced changes in CA uptake kinetics, a dual vascular endothelium and hypoxia staining was performed on tumor cryosections of the mice sacrificed 3 days after HIFU. For each mouse, two sections were selected that were located either close to (section with the largest non-viable tumor fraction) or far from (section with the lowest non-viable tumor fraction) the HIFU-treated non-viable tumor region. After acetone fixation, a fluorescent dual staining was performed using an anti-mouse glucose transporter 1 (glut-1) antibody conjugated with a DyLight 650 fluorescent label ( $4 \mathrm{~h}$, room temperature, 1:150 dilution, Novus Biologicals, Littleton, CO, USA) as an intrinsic marker for hypoxia (40) and rat anti-mouse CD31 antibody (overnight, $4{ }^{\circ} \mathrm{C}, 1: 250$ dilution, clone mec 13.3, BioLegend, San Diego, CA, USA) and secondary goat anti-rat Alexa-488 antibody ( $1 \mathrm{~h}$, room temperature, 1:500 dilution, BioLegend) for vascular endothelium staining. Fluorescence microscopy images of the entire tumor sections were acquired with a Pannoramic MIDI digital slide scanner (3DHistech, Budapest, Hungary) at 31× magnification with optimal settings for each staining and equal settings for all sections. An additional fluorescence microscopy image, at the same channel as for CD31 detection, was acquired with a longer exposure time to assess autofluorescence in the sections. ROls were manually drawn to segment the non-viable and viable tumor, based on the level of autofluorescence, which has been reported as a reliable method to accurately identify heat-fixed non-viable cells (41). Adjacent NADH-diaphorase stained sections were used as a visual reference, to confirm the correct delineation of the non-viable tumor area. Only the viable tumor tissue was used for further analysis, since the cluster analysis was also only performed on perfused, likely viable, tumor pixels. The viable tumor tissue in the section with the largest nonviable fraction was referred to as "Near non-viable" and the viable tumor tissue in the mostly viable remote section was referred to as "Remote".

The vascular surface fraction in the viable tumor region was calculated by division of the total number of CD31-positive pixels by the total number of viable tumor pixels. The level of glut-1 expression was assessed by determination of the mean glut-1 intensity in the viable tumor tissue.

\section{Statistical analysis}

Data are presented as mean \pm SD. At all experimental time points, the relative tumor sizes of the HIFU-treated and nontreated tumors were statistically compared using a two-sided $t$-test assuming equal variances. The non-perfused tumor volumes at the different experimental time points were compared for statistical significance with a two-sided paired $t$-test. Correlation analysis between the MRI-derived non-perfused tumor fractions and histology-derived non-viable tumor fractions was performed for all groups by calculation of the Pearson correlation coefficient. In 
addition, the one-to-one correspondence between the non-viable and non-perfused tumor fractions was determined by calculation of the $R^{2}$ of the data points to the line of identity. The difference between non-viable tumor fractions of the HIFU-treated and non-treated control tumors at the last experimental time point was tested for significance with a two-sided $t$-test assuming equal variances. A one-sided paired $t$-test was performed for each cluster to assess if the fraction of pixels in that cluster was increased after HIFU. The median minimal distances to the non-perfused volume of the different clusters were statistically compared using a two-sided paired $t$-test. Histological differences in vascular surface fraction and mean glut-1 intensity were tested for significance with a two-sided paired $t$-test. For all tests, the level of significance was set to $\alpha=0.05$.

\section{RESULTS}

Partial tumor ablation with HIFU resulted in a significantly lower relative tumor size on day 3 compared with the control mice (Fig. 1). Representative pharmacokinetic parameter maps in the tumor tissue before and at both time points after HIFU treatment are shown in Figure 2(A) (for the same animal). Before HIFU treatment, the tumor vasculature was characterized by higher $K^{\text {trans }}$ values in the tumor rim than in the tumor center. Furthermore, a small region of low $K^{\text {trans }}$ was observed in the tumor core. The $v_{\mathrm{e}}$ values were also generally higher in the tumor rim than in the center. Directly after partial HIFU treatment of the tumors, a large area of decreased $K^{\text {trans }}$ emerged. Such an area of decreased $K^{\text {trans }}$ was also present 3 days after HIFU treatment. At both time points after HIFU treatment, the area of decreased $K^{\text {trans }}$ corresponded to regions with low $v_{\mathrm{e}}$ and small subregions of high $v_{\mathrm{e}}$. No substantial differences in $K^{\text {trans }}$ or $v_{\mathrm{e}}$ were detected for the non-treated control animals between measurement day -1 and day 0 , although a reduction in $K^{\text {trans }}$ was observed on day 3 (Fig. 2(B)).

To assess the effect of partial ablation on tumor perfusion, non-perfused tumor volumes were determined first, based on the level of contrast enhancement in the tumor pixels. The

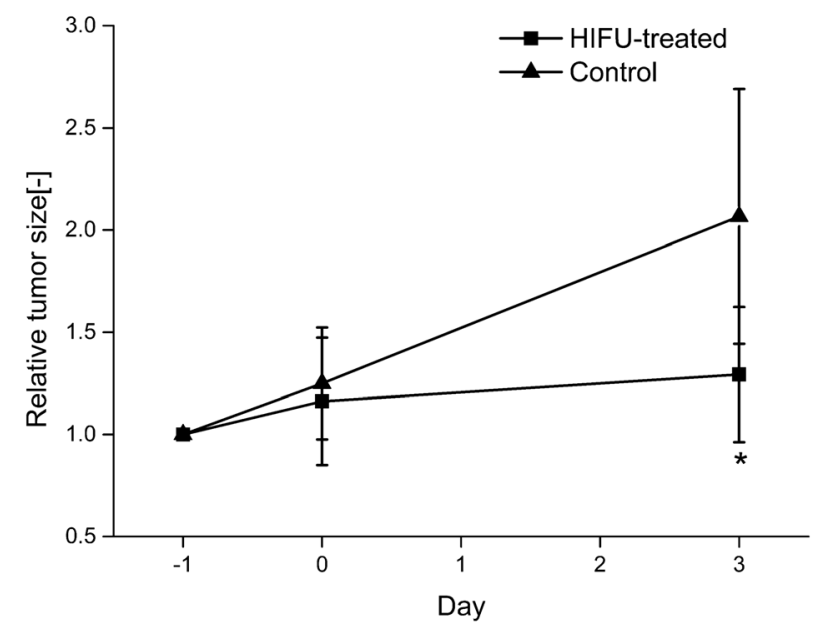

Figure 1. Tumor growth. Mean \pm SD relative tumor size, based on ROls drawn on the anatomical MRI images, at the different experimental time points for the HIFU-treated and the control animals. For the HIFU-treated animals day 0 corresponds to the day of treatment. * Significant difference in relative tumor size between the HIFU-treated and control animals on measurement day 3 (two-sided $t$-test, $p<0.05$ ). average non-perfused tumor volumes of the HIFU-treated and control animals at the different experimental time points are displayed in Figure 3(A) and (B), respectively. Directly after HIFU treatment, the non-perfused tumor volume significantly increased compared with before HIFU treatment. No significant difference between the non-perfused tumor volume 3 days after HIFU compared with either before or directly after HIFU was observed. For the control animals, the non-perfused tumor volume remained constant between measurement days -1 and 0 , whereas the non-perfused tumor volume was significantly increased on day 3.

The average histology-derived non-viable tumor fraction at the last experimental time point was higher for the HIFU-treated animals than for the control animals, although this difference was not significant (Fig. 3(C)). For the HIFU-treated animals sacrificed directly after HIFU, a high, significant correlation ( $r=$ 0.866) was observed between the MRI-derived non-perfused and histology-derived non-viable tumor fractions (Fig. 3(D)). However, the non-perfused tumor fractions were generally higher than the non-viable tumor fractions at that time point after HIFU treatment, resulting in a relatively low one-to-one correspondence $\left(R^{2}\right.$ to line of identity $\left.=0.74\right)$. At 3 days after HIFU treatment, a high correlation $(r=0.933)$ was also observed between the non-perfused and non-viable tumor fractions. At this time point after HIFU treatment, a good one-to-one correspondence between the non-perfused and non-viable tumor fractions was observed $\left(R^{2}=0.91\right)$. For the control animals, both the correlation $(r=0.242)$ and one-to-one correspondence $\left(R^{2}=0.57\right)$ between the non-perfused and non-viable tumor volume fractions were low.

In addition to the above measurements of non-perfused tumor fractions and volumes, a detailed analysis of the pharmacokinetic parameters in the perfused tumor surrounding the non-perfused ablation volume was performed. Average distributions of $K^{\text {trans }}$ and $v_{\mathrm{e}}$ in the perfused pixels of the different experimental groups are shown in the supplementary information (Fig. S2).

Cluster analysis was performed on the perfused pixels of all tumors and all time points to identify subregions with different CA uptake kinetics. Four appeared to be the minimal number of clusters with which the most prominent vascular changes could be identified, and was therefore considered as the optimal number of clusters (supplementary information Fig. S3). In Figure 4 the results of the cluster analysis with four clusters are overlaid on the tumor pixels of the same slice from the same animal as shown in Figure 2. After HIFU treatment, the number of pixels assigned to the blue and green clusters strongly decreased. The DCE-MRI curves in these clusters were characterized by a rapid upslope, a clear wash-out and substantial CA influx. At both time points after HIFU, a large region of non-perfused tissue (white pixels) emerged. Curves in the non-perfused tumor area, shown on the right of the figure, were characterized by a lack of CA inflow. The area around the non-perfused volume consisted mostly of pixels assigned to the red and yellow clusters. Contrast agent uptake in pixels assigned to the red cluster was low and relatively slow. The curves in the yellow cluster typically indicated slow uptake kinetics, but pronounced CA influx. The area of non-perfused pixels visually corresponded well to the area of non-viable tissue on the NADH-diaphorase stained section obtained 3 days after HIFU treatment at approximately the same location in the tumor, in agreement with the data presented in Figure 3(D). 
A

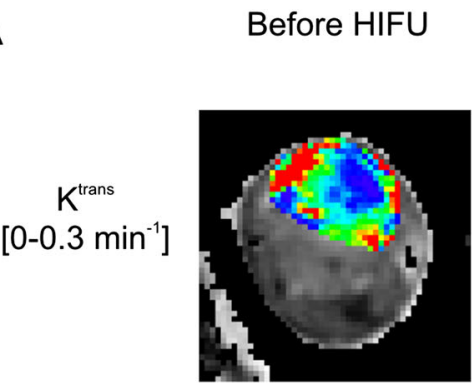

$v_{e}$ $[0-0.5]$

B
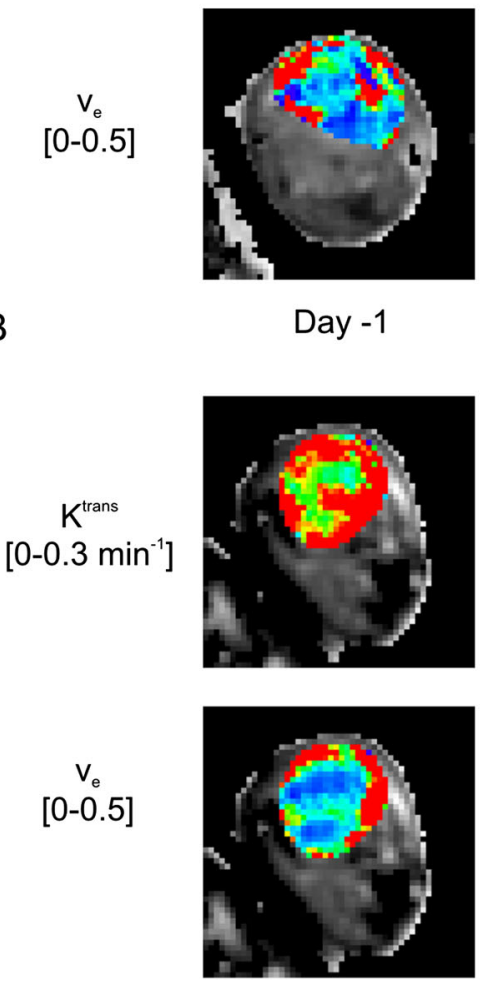

Day -1
Directly after HIFU
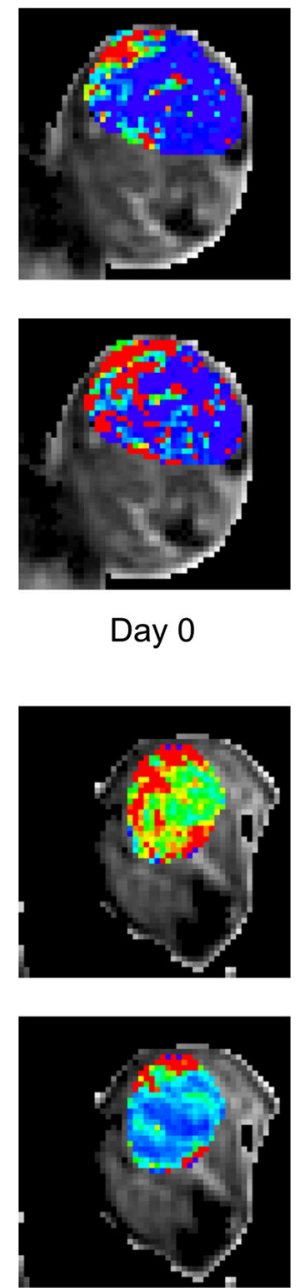

3 days After HIFU

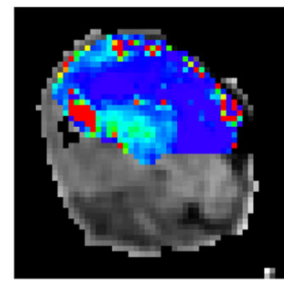

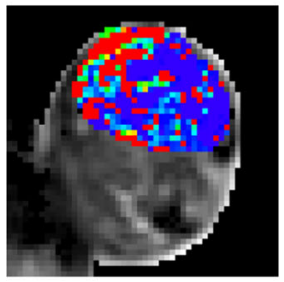

Day 0

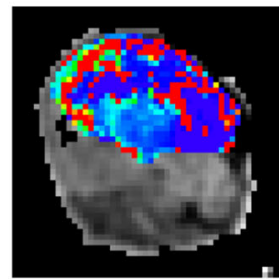

Day 3

Figure 2. Pharmacokinetic parameter maps. (A) Representative pharmacokinetic parameter maps overlaid on the tumor pixels of an axial $T_{2}$-weighted image of a tumor-bearing paw before, directly after and 3 days after HIFU treatment. (B) Representative pharmacokinetic parameter maps for a control animal at the corresponding experimental time points. The parameters were scaled according to the color bar shown on the right-hand side of the figure. The corresponding parameter range for this scale bar is indicated on the left-hand side of the parameter maps.

In Figure 5 the average fraction of pixels assigned to the four clusters is shown for the HIFU-treated animals at the different time points. Directly after HIFU treatment, the fraction of pixels in the yellow and red clusters was significantly increased compared with before HIFU. 3 days after HIFU treatment, the fraction of pixels in these clusters was not significantly higher than before HIFU. Because of the HIFU-induced increase in the fraction of pixels in the yellow and red clusters, changes in these clusters were considered as treatment associated.

In Table 1 the $K^{\text {trans }}$ and $v_{\mathrm{e}}$ parameter values in the different clusters are displayed. In the clusters with a treatment-associated increase in fraction of pixels (yellow and red clusters), $K^{\text {trans }}$ was lower than in the other (green and blue) clusters. $v_{\mathrm{e}}$ in these clusters was either higher (yellow cluster) or lower (red cluster) than in the other clusters.

To gain insight into the regional distribution of the different clusters with respect to the non-perfused volume, the minimal distance from each perfused pixel in each cluster to the closest non-perfused pixel was determined. The histograms in Figure 6 show the distribution of these minimal distances for all clusters at the two time points after HIFU treatment, and provide information on the proximity of the different clusters to the nonperfused volume. Both directly and 3 days after HIFU treatment, the red cluster was located closest to the non-perfused volume. The yellow cluster was located closer to the non-perfused volume than the green and blue clusters, but further away from the non-perfused volume than the red cluster. The median minimal distances between the four clusters and the nonperfused volume, averaged for all HIFU-treated animals, are given in Table 2 for both time points after treatment. Directly after HIFU, the average distance to the non-perfused volume was significantly smaller for the red cluster compared with all other clusters. Furthermore, a significantly smaller distance to the non-perfused volume was observed for the yellow cluster compared with the blue cluster. At 3 days after HIFU treatment, the red cluster was still significantly closer to the non-perfused volume than the blue cluster.

Representative microscopy images of H\&E-stained sections of a tumor excised at $\sim 2.5 \mathrm{~h}$ after HIFU treatment are shown in Figure 7. A region of viable tumor tissue was observed, which 


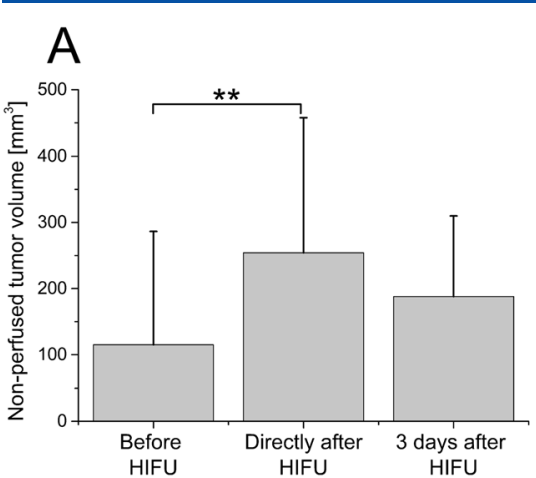

D HIFU-treated animals

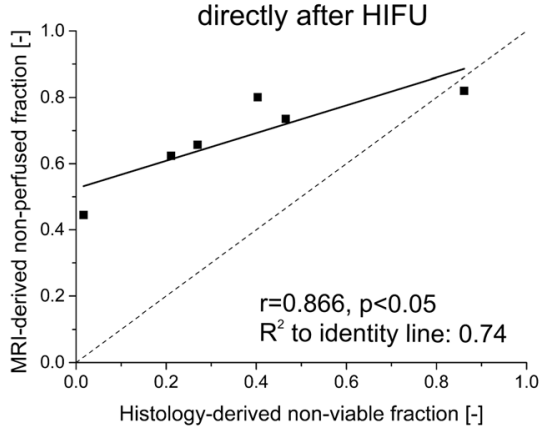

B

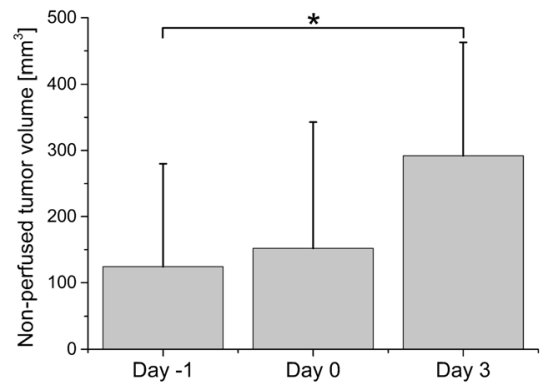

HIFU-treated animals 3 days after HIFU

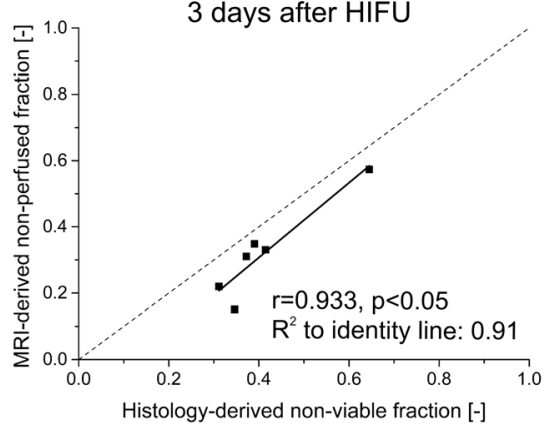

C

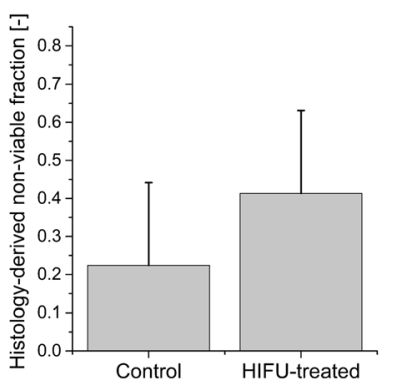

Non-treated control animals

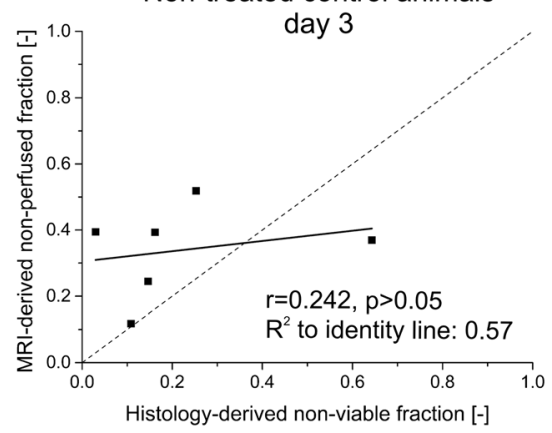

Figure 3. Non-perfused tumor volumes. (A) Mean \pm SD non-perfused tumor volumes before, directly after and 3 days after HIFU treatment. ${ }^{*}$ Significant increase in the non-perfused tumor volume directly after HIFU compared with before HIFU (two-sided paired $t$-test, $p<0.001$ ). (B) Mean \pm SD nonperfused tumor volumes on measurement days $-1,0$ and 3 for the control animals.* Significant increase in the non-perfused tumor volumes between measurement days -1 and 3 (two-sided paired $t$-test, $p<0.05$ ). (C) Mean \pm SD histology-derived non-viable tumor fractions of the control and HIFUtreated animals on measurement day 3. (D) Correlation plots of MRI-derived non-perfused tumor fractions versus histology-derived non-viable tumor fractions for the three different experimental groups. The line of identity is shown as a visual reference. The correlation coefficient and the $R^{2}$ to the line of identity are displayed in the bottom right corner of each panel.

was structurally intact (Fig. 7(A)). The cells in the viable tumor tissue were densely packed and cell nuclei had a normal appearance. In addition, a zone of coagulated tissue was observed, surrounded by a region characterized by structural disruption (Fig. 7(B)). The nuclei of the cells in the coagulated zone were typically dark and shrunken. Gaps between the tumor cells were visible in the structurally disrupted tumor tissue (Fig. 7(B), (C)). Disrupted tumor blood vessels and the presence of red blood cells in the extravascular space were observed, indicative of hemorrhages (Fig. 7(B), (D)). These hemorrhages were mainly located close to the coagulated areas.

Analysis of microvascular functionality, by a combined CD31 and glut-1 staining, was focused on the viable tumor tissue of tumors excised 3 days after HIFU. The good agreement between nonperfused and non-viable tumor fractions at that time point (Fig. 3(D)) allowed for comparison of the cluster results of the perfused pixels with changes in CD31 and glut-1 staining in the viable tumor tissue. Representative CD31 and glut-1 microscopy images of a tumor excised 3 days after HIFU are shown in Figure 8(B), with the corresponding NADH-diaphorase staining shown in Figure $8(A)$. Generally, a larger number of CD31-positive tumor pixels and a higher glut-1 intensity were observed in the "Near non-viable" tumor tissue than in the "Remote" tumor tissue. Quantitative analysis (Fig. 8(C)) showed that the average vascular surface fraction was slightly higher in the "Near non-viable" tumor tissue than in the "Remote" tumor, although these differences were not significant $(p=0.32)$. The mean glut- 1 intensity was higher (not significantly, $p=0.16$ ) in the "Near non-viable" tumor tissue compared with the "Remote" tumor tissue.

\section{DISCUSSION}

In this study, pharmacokinetic analysis of DCE-MRI data was performed to identify tumor regions with different CA uptake kinetics longitudinally after HIFU treatment. In addition to the commonly performed assessment of non-perfused volumes, cluster analysis of pharmacokinetic parameters was performed to gain insight into more subtle underlying changes in vascular status in the area surrounding the non-perfused central ablation volume.

A significant increase in the non-perfused tumor volume was observed directly after HIFU treatment (Fig. 3(A)). At this time point, the non-perfused tumor fractions were generally higher than the histology derived non-viable tumor fractions (Fig. 3(D)). This finding is possibly caused by HIFU-induced destruction of tumor vasculature in the zone surrounding the ablated region, which could lead to delayed cell death $(23,25,27,42)$. This delayed cell death may explain the better agreement between the non-viable and non-perfused tumor fractions 3 days after treatment. Temporary HIFU-induced vascular constriction (43) could also have contributed to the higher non-perfused fraction directly after treatment, although this contribution is probably minor since the non-perfused tumor volume 3 days after HIFU was not significantly lower than directly after HIFU (Fig. 3(A)). The correlation between non-viable and non-perfused tumor fractions for the 
Before

HIFU
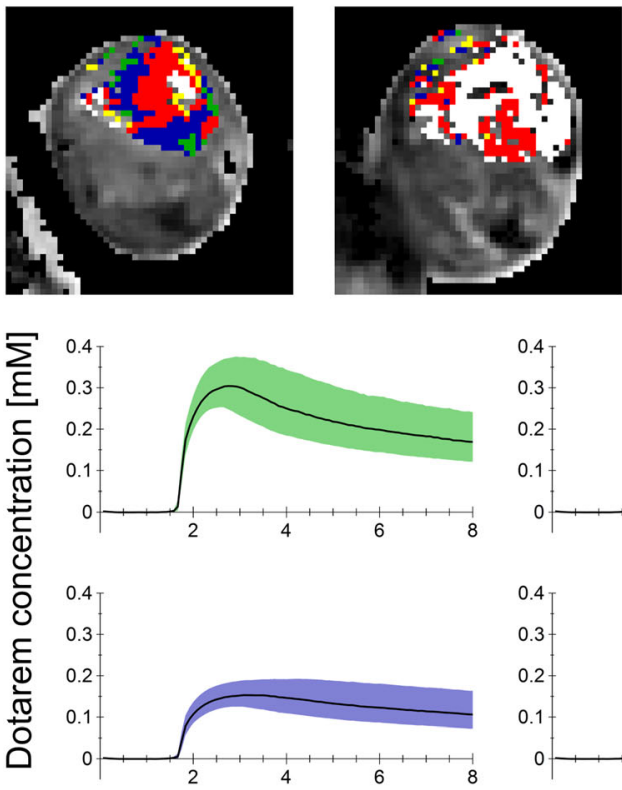

3 days after HIFU

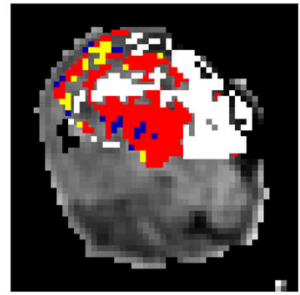

Histology 3 days after HIFU
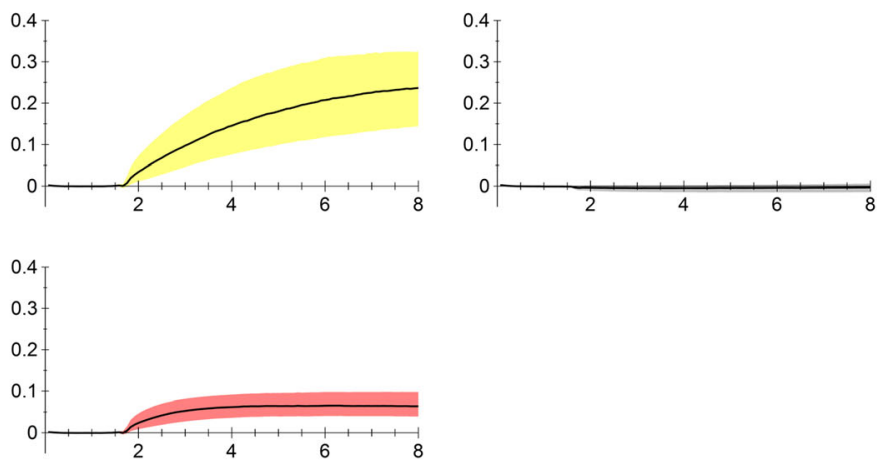

Time [min]

Figure 4. Clustering of pharmacokinetic parameter values. Representative clustering results overlaid on the tumor pixels of the $T_{2}$-weighted image of the tumor-bearing paw of the same animal as shown in Figure 2 before, directly after and 3 days after HIFU treatment. The four clusters are represented by the different colors (yellow, green, red and blue). The non-perfused pixels, which were excluded from cluster analysis, are represented by the white color. An NADH-diaphorase stained tumor section obtained at approximately the same location as the MRI slice 3 days after HIFU treatment is shown in the top right corner of the figure. A clear pale, non-viable tumor region was observed next to NADH-diaphorase-positive, viable tumor tissue. The median dynamic [CA] curves of all pixels in each of the four clusters (shown by the different colors) and the non-perfused pixels are shown at the bottom of the figure (top to bottom, left to right: green cluster, yellow cluster, non-perfused pixels, blue cluster and red cluster). The bands around the curves represent the range between the 25 th and 75 th percentiles.

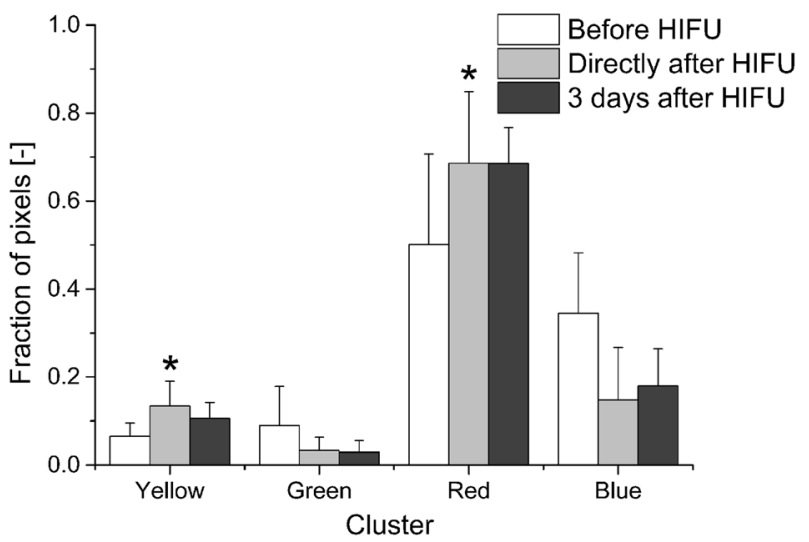

Figure 5. Cluster composition at the different experimental time points. Mean \pm SD fraction of pixels assigned to the four different clusters before, directly after and 3 days after HIFU treatment. * Significant increase in the fraction of pixels directly after HIFU compared with before HIFU ( $p<0.05$, one-sided paired $t$-test).

Table 1. $K^{\text {trans }}$ and $v_{\mathrm{e}}$ values (mean $\pm \mathrm{SD}$ ) in the four clusters

\begin{tabular}{lcc} 
Cluster & $K^{\text {trans }}\left[\mathrm{min}^{-1}\right]$ & $v_{\mathrm{e}}[-]$ \\
\hline Yellow & $0.066 \pm 0.054$ & $0.495 \pm 0.170$ \\
Green & $0.356 \pm 0.107$ & $0.223 \pm 0.098$ \\
Red & $0.039 \pm 0.026$ & $0.095 \pm 0.061$ \\
Blue & $0.150 \pm 0.044$ & $0.138 \pm 0.067$ \\
\hline
\end{tabular}

non-treated control animals was low, in contrast to the treated animals (Fig. 3(D)). For the control animals, the non-perfused tumor fraction was generally higher than the non-viable tumor fraction. Likely, some of the non-perfused pixels represented hypoxic, yet viable, tumor tissue, which is also known to be poorly perfused $(44,45)$. This hypoxic tissue may become necrotic at a later stage of tumor progression. Therefore, nonperfused pixels do not necessarily represent necrosis, leading to a disagreement between the non-viable and non-perfused tumor fractions. This complicates the classification of tumor as non-viable solely based on the lack of contrast enhancement after HIFU in a setting in which tumors were already relatively non-perfused before treatment.

Nevertheless, pharmacokinetic analysis can still give additional insights into uptake kinetics and thereby provide information on changes in the underlying vascular status in the zone peripheral to the central ablation volume. Histogram and cluster analysis (Figures S2, 4 and 5) of the pharmacokinetic parameters in the perfused pixels showed that there were subtle yet significant HIFU-induced changes in the pharmacokinetic parameter values in the areas surrounding the non-perfused volume. Since in two clusters the fraction of pixels significantly increased directly after HIFU treatment (Fig. 5), these alterations were assumed to be treatment associated. The composition of the clusters was very similar directly and 3 days after HIFU treatment, which indicated that there were no apparent changes in CA uptake kinetics in the perfused tumor tissue between these time points. One of the treatment-associated clusters was characterized by a low $K^{\text {trans }}$ 

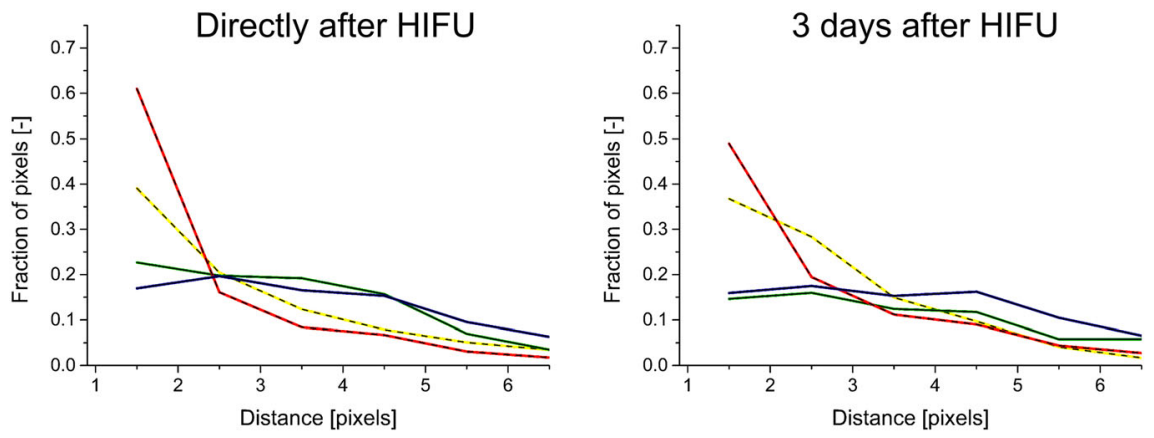

Figure 6. Spatial distribution of clusters with respect to non-perfused volume. Distribution of the minimal distance from the pixels in each of the four different clusters to the closest non-perfused pixel directly after and 3 days after HIFU treatment. The yellow, green, red and blue lines represent the different clusters. The dashed lines represent the clusters with a treatment-associated increase in the fraction of pixels (yellow and red clusters) and the solid lines represent the other (blue and green) clusters.

Table 2. Distances from clusters to the non-perfused volume. Median minimal distance from the different clusters to the non-perfused volume averaged for all HIFU-treated animals, at both time points after HIFU treatment. Data are presented as mean \pm SD. \# , " Significantly smaller distance from a cluster to the non-perfused volume compared with the cluster(s) indicated between the parentheses, with $p<0.05$ and $p<0.001$, respectively.

\begin{tabular}{|lll|} 
Cluster & \multicolumn{1}{c}{$\begin{array}{c}\text { Distance directly } \\
\text { after HIFU [pixels] }\end{array}$} & $\begin{array}{c}\text { Distance 3 days } \\
\text { after HIFU [pixels] }\end{array}$ \\
\hline Yellow & $2.31 \pm 0.67^{\text {\#(blue) }}$ & $2.51 \pm 0.34$ \\
Green & $2.79 \pm 1.67$ & $3.67 \pm 1.78$ \\
Red & $1.52 \pm 0.51^{\text {\#(green, blue) \#\#(yellow) }}$ & $2.15 \pm 0.68$ \#(blue) \\
Blue & $3.13 \pm 1.50$ & $3.41 \pm 1.25$ \\
\hline
\end{tabular}
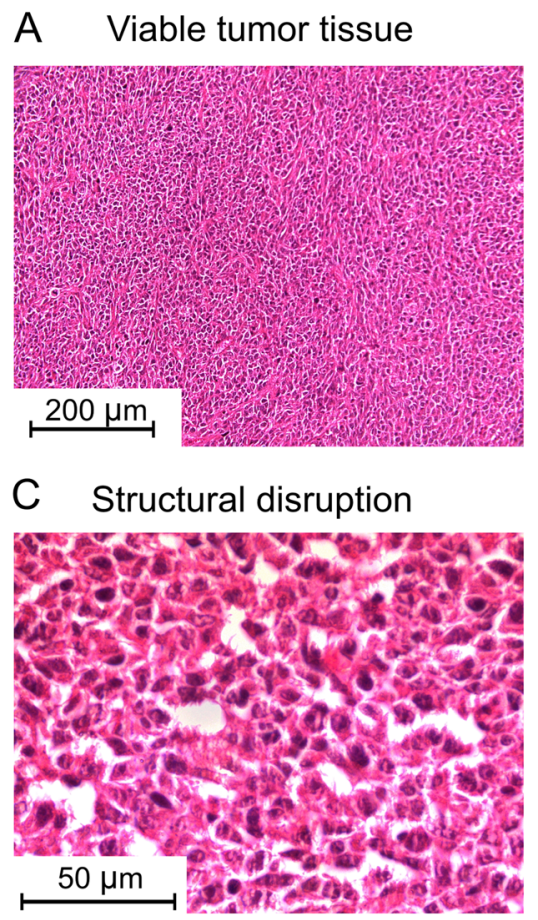

and high $v_{\mathrm{e}}$ (yellow cluster) and the other by a low $K^{\text {trans }}$ and low $v_{\mathrm{e}}$ (red cluster) combination (Table 1). The uptake kinetics (Fig. 4) characterizing the region with low $K^{\text {trans }}$ and high $v_{\mathrm{e}}$ could be explained by a more difficult CA access due to HIFU-induced vascular congestion and hemorrhage, but a higher capacity for accumulation due to the presence of vacuolation and structural disruption in the extravascular tissue space. The low $K^{\text {trans }}$ and low $v_{\mathrm{e}}$ in the other cluster with a treatment-associated increase in fraction of pixels (red cluster) was likely caused by more severe vascular damage, which may include vascular disruption, congestion and hemorrhage. Regions with these types of vascular characteristic around the central region of coagulative necrosis were previously also identified with DCE-MRI analysis and confirmed by extensive histological evaluation in a study on HIFU treatment of rabbit thigh muscle (31).

Pixels in the clusters with treatment-associated changes (yellow and red clusters) were located closer to the non-perfused

\section{B Treatment border zone}
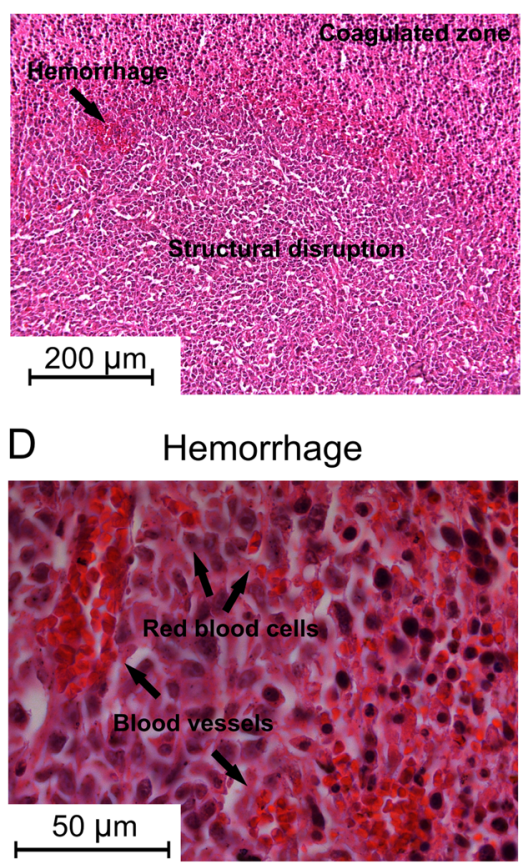

Figure 7. H\&E microscopy. Representative brightfield microscopy images of H\&E-stained sections of a HIFU-treated CT26.WT tumor excised at 2.5 h after treatment. (A) Viable tumor tissue at 20x magnification. (B) Border zone of coagulated tumor tissue surrounded by structurally disrupted tumor

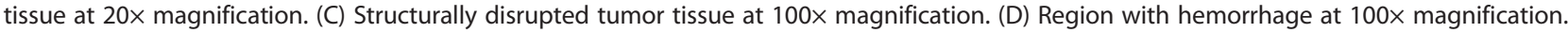


A

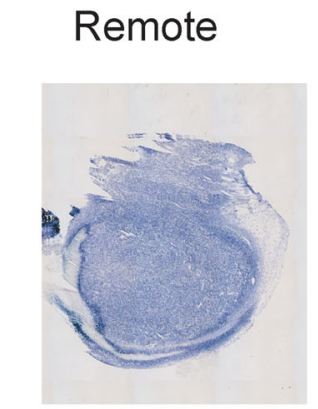

Near non-viable

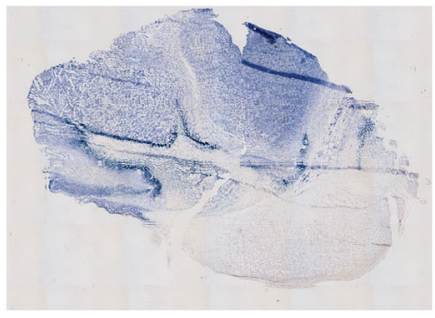

B

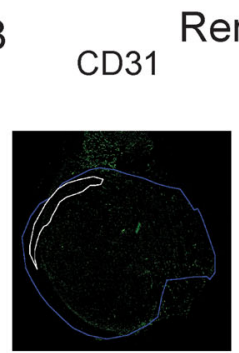

\section{Rer}

emote

Glut-1

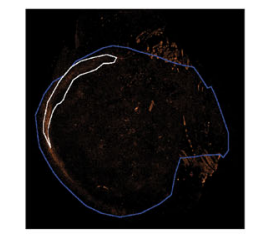

Near non-viable

CD31

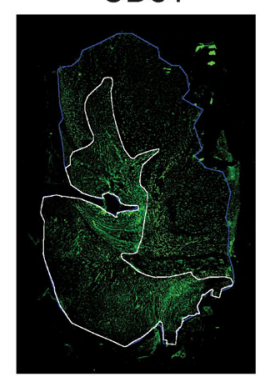

Glut-1

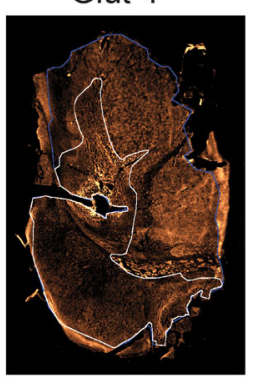

C
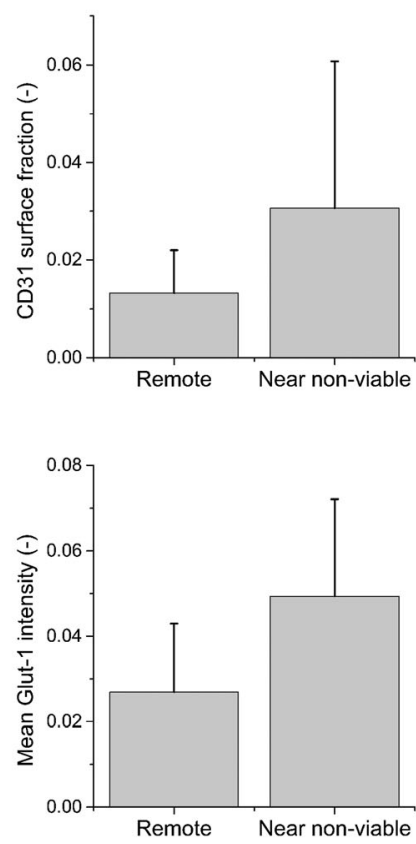

Figure 8. Microvascular endothelium (CD31) and hypoxia (glut-1) immunohistochemistry. (A) NADH-diaphorase staining for cell viability of a section with a large non-viable tumor fraction (with "Near non-viable" tumor tissue) and a distant section with a low non-viable tumor fraction (with "Remote" tumor tissue). Both sections originate from the same tumor, excised 3 days after HIFU. (B) Representative CD31 and glut-1 fluorescence microscopy images of adjacent tumor sections. The non-viable and entire tumor tissue are delineated in white and blue, respectively. The analysis was performed on the viable (either "Remote" or "Near non-viable") tumor tissue. (C) Quantification of the mean \pm SD microvascular density and mean \pm SD glut-1 intensity in the "Remote" and "Near non-viable" tumor tissue of the "3 days after HIFU" animals.

pixels than pixels in the other clusters (Fig. 6). In addition, H\&E staining confirmed the presence of tumor regions with structural disruption and hemorrhage after HIFU (Fig. 7). Vascular endothelium (CD31) and hypoxia (glut-1) dual staining was performed to gain more insight into the cause and consequences of the treatment-associated changes in the perfused tumor pixels. Since the pixels in the clusters with treatment-associated changes (yellow and red clusters) were located closer to the non-perfused pixels than pixels in the other clusters (Fig. 6), a section close to the non-perfused tumor and a remote section were selected for these histological analyses. No decreased vascular density could be observed in the "Near non-viable" tumor tissue compared with the "Remote" tumor tissue (Fig. 8). This suggested that the increased fraction of pixels in the red and yellow clusters, both characterized by a low $K^{\text {trans }}$, was mainly a result of a decreased vascular function rather than a reduction of microvascular density. The decreased vascular function was also suggested by the higher glut- 1 intensity in these regions. These findings were in agreement with the observed structural disruption and hemorrhage on H\&E staining (Fig. 7). Furthermore, the higher glut-1 intensity indicated that the observed treatmentassociated changes in vascular status may have consequences for tumor oxygenation. Association of low $K^{\text {trans }}$ and $v_{\mathrm{e}}$ values in non-necrotic tumor regions with hypoxic tumor tissue has been previously shown by Egeland et al. (46).

In our analyses, the non-perfused volume was defined based on the level of signal enhancement, and subsequently these non-perfused pixels were excluded from clustering. This approach was employed since tracer-kinetic parameter estimation could typically not be reliably performed for dynamic [CA] curves within this region. For these curves, model regressions were often characterized by low $R^{2}$ and tracer-kinetic parameters with non-physiological values, which has been commonly observed (47). Inclusion of such data in the clustering would negatively affect the accuracy of the clustering procedure. A potential drawback of the exclusion of the non-perfused volume could be that it may affect the voxel population that is selected for clustering at the different experimental time points. It is expected that this would mostly influence the absolute number of pixels within the different clusters and that the existence of areas with the described low $K^{\text {trans }}$ and low $v_{\mathrm{e}}$, or low $K^{\text {trans }}$ and high $v_{\mathrm{e}}$, combination in areas surrounding the non-perfused volume after HIFU remains consistent. Similarly, whereas the absolute average distance from the clusters to the non-perfused volume may be slightly affected by the definition of the non-perfused volume, it only has minor influence on the relative differences in these distances between the clusters. Therefore, the clustering can be regarded as a useful tool to identify regions with specific uptake kinetics surrounding the non-perfused volume and to determine the regional distribution of these regions in the tumor.

A potential drawback of $k$-means clustering is that it requires the $a$ priori specification of the number of clusters to be used. This number was optimized by investigating which was the minimum number of clusters to identify the most prominent changes in tracer-kinetic parameters after HIFU. Figure S3 demonstrates that the average tracer-kinetic parameter values within each cluster, as well as the significance of changes in a cluster population after treatment, are partly influenced by the number of clusters that is used in the analysis. Nevertheless, the general increase in regions with a low $K^{\text {trans }}$ and low $v_{\mathrm{e}}$, or low $K^{\text {trans }}$ and high $v_{\mathrm{e}}$, combination could be observed for various values of $k$ (i.e., $k=4-6$ ). Although the observed changes in the 
fractions of pixels in the different clusters were induced by HIFU treatment and histology provided information on the causes and consequences of these changes, the kinetic uptake characteristics of the red and yellow clusters cannot be regarded as specific for HIFU-treated tumor based on the presented results. The relatively high fraction of pixels in the red cluster before treatment (Fig. 5) indicates that pixels with these characteristics can be naturally present in this tumor model. This could be explained by the fact that for this tumor model perfusion was relatively low in the tumor center. Therefore, most likely hypoxic regions with an impaired perfusion are naturally present. The large extent of poorly perfused tumor tissue in the non-treated tumors was rather unexpected, because it has been previously described that subcutaneous CT26 tumors are generally well vascularized (48) with limited necrosis (49). The overall lower perfusion observed in the present study could be caused by the difference in inoculation site (hind limb in our study versus back in the referred studies). Application of the cluster analysis in better perfused tumor models could potentially allow for the detection of more pronounced HIFU-induced changes in CA uptake kinetics and clusters that are more specific for HIFU-induced vascular alterations.

Additional histological analysis, in which the clusters with specific vascular characteristics are spatially compared with histological features, could give further insights into the underlying effects causing the observed vascular changes. The use of MRI-visible and histology-visible fiducial markers or tissue-marking dyes could aid in retrieving the MRI slice orientation on histology and thereby enable more direct histological validation of the cluster results (50).

Furthermore, the histological analysis could be extended in order to allow for a more complete assessment of vascular status after HIFU treatment. The combined glut-1 and CD31 staining represents a relatively indirect evaluation of vascular function and is therefore probably not sensitive enough to spatially differentiate between the different clusters. Infusion of the fluorescent vascular perfusion marker Hoechst (51), or vascular permeability marker Evans Blue (52), would allow for a more direct and complete assessment of the tumor vascular characteristics and altered tumor vascular status after HIFU treatment.

HIFU treatment was performed outside the MR system, since the preclinical therapeutic ultrasound transducer is not MR compatible. In a HIFU set-up with MRI guidance, temperature maps could be acquired during the treatment, allowing changes in the pharmacokinetic parameters to be compared with the applied thermal dose (31). This information would also allow for a better definition of the danger zones around the central ablation volumes and a more detailed comparison between applied thermal dose, temperature-dependent changes in uptake kinetics and histological appearance of these regions.

Measurements made longitudinally during a longer time period after HIFU treatment would give further insight into the dynamic response of tumor tissue to HIFU treatment. The DCE-MRI data in the current study were acquired up to 3 days after treatment. At a later time point after HIFU, repair processes such as regeneration, fibrosis and revascularization are expected, which could alter the pharmacokinetic parameter values (26). In addition, it would be of interest whether early changes in CA uptake kinetics can be of prognostic value for the ultimate fate of the tissue.

In conclusion, it was demonstrated that analysis of DCE-MRI data by means of cluster analysis on pharmacokinetic parameters can be used to identify regions with different CA uptake kinetics after HIFU treatment, which may be reflective of their vascular status. In addition to the commonly performed analysis of nonperfused tumor volume after HIFU treatment, the presented methodology gives additional insight into uptake kinetics in the transition zone adjacent to central ablation volume. This method could aid in assessment of the consequences of the different vascular alterations after HIFU for the microenvironment and fate of the tissue and the sensitivity of the tumor to additional therapies.

\section{Acknowledgements}

The authors acknowledge Ralf Seip from Philips Research for his advice on the HIFU treatment settings.

This research was performed within the framework of CTMM, the Center for Translational Molecular Medicine (www.ctmm.nl), project VOLTA (grant 05T-201). The authors have no conflicts of interest.

\section{REFERENCES}

1. Tempany CM, McDannold NJ, Hynynen K, Jolesz FA. Focused ultrasound surgery in oncology: overview and principles. Radiology $2011 ; 259(1): 39-56$.

2. Hesley GK, Gorny KR, Woodrum DA. MR-guided focused ultrasound for the treatment of uterine fibroids. Cardiovasc. Interv. Radiol. 2013; 36(1): 5-13.

3. Voogt MJ, Trillaud H, Kim YS, Mali WP, Barkhausen J, Bartels LW, Deckers R, Frulio N, Rhim H, Lim HK, Eckey T, Nieminen HJ, Mougenot C, Keserci B, Soini J, Vaara T, Kohler MO, Sokka S, van den Bosch MA. Volumetric feedback ablation of uterine fibroids using magnetic resonance-guided high intensity focused ultrasound therapy. Eur. Radiol. 2012; 22(2): 411-417.

4. Napoli A, Anzidei M, Ciolina F, Marotta E, Cavallo Marincola B, Brachetti G, Di Mare L, Cartocci G, Boni F, Noce V, Bertaccini L, Catalano C. MR-guided high-intensity focused ultrasound: current status of an emerging technology. Cardiovasc. Interv. Radiol. 2013; 36(5): 1190-1203.

5. Schmitz AC, Gianfelice D, Daniel BL, Mali WP, van den Bosch MA. Image-guided focused ultrasound ablation of breast cancer: current status, challenges, and future directions. Eur. Radiol. 2008; 18(7): 1431-1441.

6. Merckel LG, Bartels LW, Kohler MO, van den Bongard HJ, Deckers R, Mali WP, Binkert CA, Moonen CT, Gilhuijs KG, van den Bosch MA. MR-guided high-intensity focused ultrasound ablation of breast cancer with a dedicated breast platform. Cardiovasc. Interv. Radiol. 2013; 36(2): 292-301.

7. Murat FJ, Poissonnier L, Pasticier G, Gelet A. High-intensity focused ultrasound (HIFU) for prostate cancer. Cancer Control 2007; 14(3): 244-249.

8. Rouviere O, Girouin N, Glas L, Ben Cheikh A, Gelet A, MegeLechevallier F, Rabilloud M, Chapelon JY, Lyonnet D. Prostate cancer transrectal HIFU ablation: detection of local recurrences using T2weighted and dynamic contrast-enhanced MRI. Eur. Radiol. 2010; 20(1): 48-55.

9. Kirkham AP, Emberton M, Hoh IM, Illing RO, Freeman AA, Allen C. MR imaging of prostate after treatment with high-intensity focused ultrasound. Radiology 2008; 246(3): 833-844.

10. Rouviere O, Gelet A, Crouzet S, Chapelon JY. Prostate focused ultrasound focal therapy - imaging for the future. Nat. Rev. Clin. Oncol. 2012; 9(12): 721-727.

11. Leslie T, Ritchie R, Illing R, Ter Haar G, Phillips R, Middleton M, Bch B, Wu F, Cranston D. High-intensity focused ultrasound treatment of liver tumours: post-treatment MRI correlates well with intraoperative estimates of treatment volume. Br. J. Radiol. 2012; 85(1018): 1363-1370.

12. Wijlemans JW, Bartels LW, Deckers R, Ries M, Mali WP, Moonen CT, van den Bosch MA. Magnetic resonance-guided high-intensity focused ultrasound (MR-HIFU) ablation of liver tumours. Cancer Imaging 2012; 12: 387-394. 
13. Leslie TA, Kennedy JE, Illing RO, Ter Haar GR, Wu F, Phillips RR, Friend PJ, Roberts IS, Cranston DW, Middleton MR. High-intensity focused ultrasound ablation of liver tumours: can radiological assessment predict the histological response? Br. J. Radiol. 2008; 81(967): 564-571.

14. Kennedy JE. High-intensity focused ultrasound in the treatment of solid tumours. Nat. Rev. Cancer 2005; 5(4): 321-327.

15. Hynynen K. MRl-guided focused ultrasound treatments. Ultrasonics 2010; 50(2): 221-229.

16. ter Haar G. Therapeutic applications of ultrasound. Prog. Biophys. Mol. Biol. 2007; 93(1-3): 111-129.

17. Kohler MO, Mougenot C, Quesson B, Enholm J, Le Bail B, Laurent C, Moonen CT, Ehnholm GJ. Volumetric HIFU ablation under 3D guidance of rapid MRI thermometry. Med. Phys. 2009; 36(8): 3521-3535.

18. Ishihara Y, Calderon A, Watanabe H, Okamoto K, Suzuki Y, Kuroda K, Suzuki Y. A precise and fast temperature mapping using water proton chemical shift. Magn. Reson. Med. 1995; 34(6): 814-823.

19. de Senneville BD, Mougenot C, Quesson B, Dragonu I, Grenier N, Moonen CT. MR thermometry for monitoring tumor ablation. Eur. Radiol. 2007; 17(9): 2401-2410.

20. Zhou YF. High intensity focused ultrasound in clinical tumor ablation. World J. Clin. Oncol. 2011; 2(1): 8-27.

21. Venkatesan AM, Partanen A, Pulanic TK, Dreher MR, Fischer J, Zurawin RK, Muthupillai R, Sokka S, Nieminen HJ, Sinaii N, Merino $M$, Wood BJ, Stratton P. Magnetic resonance imaging-guided volumetric ablation of symptomatic leiomyomata: correlation of imaging with histology. J. Vasc. Interv. Radiol. 2012; 23(6): 786-794.

22. Kim YS, Lim HK, Kim JH, Rhim H, Park BK, Keserci B, Kohler MO, Bae DS, Kim BG, Lee JW, Kim TJ, Sokka S, Lee JH. Dynamic contrastenhanced magnetic resonance imaging predicts immediate therapeutic response of magnetic resonance-guided high-intensity focused ultrasound ablation of symptomatic uterine fibroids. Invest. Radiol. 2011; 46(10): 639-647.

23. Vaupel PW, Kelleher DK. Pathophysiological and vascular characteristics of tumours and their importance for hyperthermia: heterogeneity is the key issue. Int. J. Hyperth. 2010; 26: 211-223.

24. Kong G, Braun RD, Dewhirst MW. Characterization of the effect of hyperthermia on nanoparticle extravasation from tumor vasculature. Cancer Res. 2001; 61(7): 3027-3032.

25. Chu KF, Dupuy DE. Thermal ablation of tumours: biological mechanisms and advances in therapy. Nat. Rev. Cancer 2014; 14(3): 199-208.

26. Cheng HL, Purcell CM, Bilbao JM, Plewes DB. Usefulness of contrast kinetics for predicting and monitoring tissue changes in muscle following thermal therapy in long survival studies. J. Magn. Reson. Imaging 2004; 19(3): 329-341.

27. Luo W, Zhou X, Gong X, Zheng M, Zhang J, Guo X. Study of sequential histopathologic changes, apoptosis, and cell proliferation in rabbit livers after high-intensity focused ultrasound ablation. J. Ultrasound Med. 2007; 26(4): 477-485.

28. Khiat A, Gianfelice D, Amara M, Boulanger Y. Influence of posttreatment delay on the evaluation of the response to focused ultrasound surgery of breast cancer by dynamic contrast enhanced MRI. Br. J. Radiol. 2006; 79(940): 308-314.

29. Tofts PS, Brix G, Buckley DL, Evelhoch JL, Henderson E, Knopp MV, Larsson HB, Lee TY, Mayr NA, Parker GJ, Port RE, Taylor J, Weisskoff RM. Estimating kinetic parameters from dynamic contrastenhanced $\mathrm{T}_{1}$-weighted MRI of a diffusable tracer: standardized quantities and symbols. J. Magn. Reson. Imaging 1999; 10: 223-232.

30. Hijnen NM, Heijman E, Kohler MO, Ylihautala M, Ehnholm GJ, Simonetti AW, Grull H. Tumour hyperthermia and ablation in rats using a clinical MR-HIFU system equipped with a dedicated small animal set-up. Int. J. Hyperth. 2012; 28(2): 141-155.

31. Cheng HL, Purcell CM, Bilbao JM, Plewes DB. Prediction of subtle thermal histopathological change using a novel analysis of Gd-DTPA kinetics. J. Magn. Reson. Imaging 2003; 18(5): 585-598.

32. Seip R, Chin CT, Hall CS, Raju BI, Ghanem A, Tiemann K. Targeted ultrasound-mediated delivery of nanoparticles: on the development of a new HIFU-based therapy and imaging device. IEEE Trans. Biomed. Eng. 2010; 57(1): 61-70.

33. Hectors SJ, Jacobs I, Strijkers GJ, Nicolay K. Multiparametric MRI analysis for the identification of high intensity focused ultrasoundtreated tumor tissue. PLoS One 2014; 9(6): e99936.

34. Karlsson M, Nordell B. Phantom and in vivo study of the Look-Locker $\mathrm{T}_{1}$ mapping method. Magn. Reson. Imaging 1999; 17(10): 1481-1488.
35. Kleppesto M, Larsson C, Groote I, Salo R, Vardal J, Courivaud F Bjornerud $A$. $T_{2}{ }^{*}$-correction in dynamic contrast-enhanced MRI from double-echo acquisitions. J. Magn. Reson. Imaging 2014; 39(5): 1314-1319.

36. Yu Y, Jiang $Q$, Wang $H$, Bao $S$, Haacke EM, Hu J. Quantitative perfusion and permeability analysis of animal brain using dual echo DCE-MRI. Proceedings of the 19th Annual Meeting ISMRM, Montreal, Quebec, Canada, 2011; 2060.

37. Vander Elst L, Raynaud J-S, Vives V, Santus R, Louin G, Robert P, Port $M$, Corot C, Muller R. Comparative relaxivities and efficacies of gadolinium-based commercial contrast agents. Proceedings of the 21st Annual Meeting ISMRM, Salt Lake City, UT, USA, 2013, 746.

38. Tofts PS, Kermode AG. Measurement of the blood-brain barrier permeability and leakage space using dynamic MR imaging. 1. Fundamental concepts. Magn. Reson. Med. 1991; 17(2): 357-367.

39. Fruytier AC, Magat J, Colliez F, Jordan B, Cron G, Gallez B. Dynamic contrast-enhanced MRI in mice at high field: estimation of the arterial input function can be achieved by phase imaging. Magn. Reson. Med. 2014; 71(2): 544-550.

40. Airley RE, Loncaster J, Raleigh JA, Harris AL, Davidson SE, Hunter RD, West CM, Stratford IJ. GLUT-1 and CAIX as intrinsic markers of hypoxia in carcinoma of the cervix: relationship to pimonidazole binding. Int. J. Cancer 2003; 104(1): 85-91.

41. Hennings L, Kaufmann Y, Griffin R, Siegel E, Novak P, Corry P, Moros EG, Shafirstein G. Dead or alive? Autofluorescence distinguishes heat-fixed from viable cells. Int. J. Hyperth. 2009; 25(5): 355-363.

42. Wu F, Chen WZ, Bai J, Zou JZ, Wang ZL, Zhu H, Wang ZB. Tumor vessel destruction resulting from high-intensity focused ultrasound in patients with solid malignancies. Ultrasound Med. Biol. 2002; 28(4): 535-542.

43. Hynynen K, Chung AH, Colucci V, Jolesz FA. Potential adverse effects of high-intensity focused ultrasound exposure on blood vessels in vivo. Ultrasound Med. Biol. 1996; 22(2): 193-201.

44. Gillies RJ, Schornack PA, Secomb TW, Raghunand N. Causes and effects of heterogeneous perfusion in tumors. Neoplasia 1999; 1(3): 197-207.

45. Tozer GM, Lewis S, Michalowski A, Aber V. The relationship between regional variations in blood-flow and histology in a transplanted rat fibrosarcoma. Br. J. Cancer 1990; 61(2): 250-257.

46. Egeland TA, Gulliksrud K, Gaustad JV, Mathiesen B, Rofstad EK. Dynamic contrast-enhanced-MRI of tumor hypoxia. Magn. Reson. Med. 2012; 67(2): 519-530.

47. Koh TS, Thng CH, Hartono S, Dominguez LT, Lim TK, Huynh $\mathrm{H}$, Martarello L, Ng QS. Assessment of tumor necrotic fraction by dynamic contrast-enhanced MRI: a preclinical study of human tumor xenografts with histopathologic correlation. NMR Biomed. 2014; 27 (4): 486-494.

48. Ogawara K, Un K, Minato K, Tanaka K, Higaki K, Kimura T. Determinants for in vivo anti-tumor effects of PEG liposomal doxorubicin: importance of vascular permeability within tumors. Int. J. Pharm. 2008; 359(1/2): 234-240.

49. Aulino P, Berardi E, Cardillo VM, Rizzuto E, Perniconi B, Ramina C, Padula F, Spugnini EP, Baldi A, Faiola F, Adamo S, Coletti D. Molecular, cellular and physiological characterization of the cancer cachexia-inducing C26 colon carcinoma in mouse. BMC Cancer 2010; 10: 363.

50. McGrath DM, Vlad RM, Foltz WD, Brock KK. Technical note: Fiducial markers for correlation of whole-specimen histopathology with MR imaging at 7 tesla. Med. Phys. 2010; 37(5): 2321-2328.

51. van der Sanden BP, Rozijn TH, Rijken PF, Peters HP, Heerschap A, van der Kogel AJ, Bovee WM. Noninvasive assessment of the functional neovasculature in $9 \mathrm{~L}$-glioma growing in rat brain by dynamic ${ }^{1} \mathrm{H}$ magnetic resonance imaging of gadolinium uptake. J. Cereb. Blood Flow Metab. 2000; 20(5): 861-870.

52. Yang FY, Ko CE, Huang SY, Chung IF, Chen GS. Pharmacokinetic changes induced by focused ultrasound in glioma-bearing rats as measured by dynamic contrast-enhanced MRI. PLoS One 2014; 9(3): e92910.

\section{SUPPORTING INFORMATION}

Additional supporting information can be found in the online version of this article at the publisher's website. 